\title{
Relationship between tissue lipid peroxidation and peroxidizability index after $\alpha$-linolenic, eicosapentaenoic, or docosahexaenoic acid intake in rats
}

\author{
Morio Saito $^{1 *}$ and Kazuhiro Kubo ${ }^{1,2}$ \\ ${ }^{1}$ Division of Food Science, Independent Administrative Institution, National Institute of Health and Nutrition, Shinjuku-ku, \\ Tokyo 162-8636, Japan \\ ${ }^{2}$ Japan Science and Technology Corporation, Kawaguchi, Saitama 332-0012, Japan
}

(Received 18 December 2001 - Revised 11 July 2002 - Accepted 11 August 2002)

\begin{abstract}
In a previous study, we found that the extent of dietary $n$ - 3 docosahexaenoic acid (DHA)-stimulated tissue lipid peroxidation was less than expected from the relative peroxidizability index of the total tissue lipids in rats with adequate vitamin E nutritional status. This suppression of lipid peroxidation was especially prominent in the liver. To elucidate whether this phenomenon was unique to DHA, we compared the peroxidation effects of $n-3 \alpha$-linolenic acid $(\alpha-\mathrm{LN})$ and $n-3$ eicosapentaeonic acid (EPA) with those of DHA in rats. Either $\alpha$-LN (8.6\% of total energy), EPA $(8.2 \%)$, or DHA $(8.0 \%)$ and one of two levels of dietary vitamin E $(7.5$ and $54 \mathrm{mg} / \mathrm{kg}$ diet) were fed to rats for $22 \mathrm{~d}$. Levels of conjugated diene, chemiluminescence emission and thiobarbituric acid (TBA)-reactive substance in the liver, kidney, and testis were determined as indicators of lipid peroxidation. In rats fed the DHA diet deficient in vitamin E $(7.5 \mathrm{mg} /$ $\mathrm{kg}$ diet), TBA values in the liver, kidney, and testis correlated well with the tissues' relative peroxidizability indices. In rats fed the $\alpha$-LN diet with an adequate level of vitamin $\mathrm{E}$ $(54 \mathrm{mg} / \mathrm{kg}$ diet), a close association between relative peroxidizability indices and lipid peroxide levels was observed in all the tissues analysed. However, in rats fed either the EPA diet or the DHA diet with an adequate level of vitamin E, the extent of lipid peroxidation in each tissue was less than expected from the relative peroxidizability index. This suppression was particularly marked in the liver. We concluded that suppression of lipid peroxidation below the relative peroxidizability index was not unique to DHA, but was also seen with EPA, which has five double bonds, in rats with adequate vitamin $\mathrm{E}$ nutritional status, but not with $\alpha-\mathrm{LN}$, which has three double bonds.
\end{abstract}

Docosahexaenoic acid: Eicosapentaeonic acid: Lipid peroxidation: Peroxidizability index

n-3 Eicosapentaenoic acid (EPA; $20: 5 n-3)$ and docosahexaenoic acid (DHA; $22: 6 n-3)$ are the major polyunsaturated fatty acids (PUFA) in fish oils. Some vegetable oils such as flaxseed and perilla oils are rich in another $n-3$ fatty acid, $\alpha$-linolenic acid $(\alpha-\mathrm{LN} ; 18: 3 n-3)$. Consumption of $n-3$ PUFA has been shown to be associated with a low incidence of atherosclerosis and cardiovascular diseases (Dyerberg, 1986; Herold \& Kinsella, 1986; Harris, 1989; Simopoulos, 1991, 1999; Crawford et al. 2000). Studies of non-human primates and newborn infants have also shown that DHA is essential to the normal functional development of the retina and brain, especially in premature babies (Nestel, 1990; Simopoulos, 1991). Consequently, concentrates of $n$-3 PUFA, such as EPA and DHA from fish oils in particular, have been used as medicinal and/or nutritional supplements (Drevon, 1992; Lands, 1992).

However, the ingestion of purified DHA (Saito et al. 1996; Kubo et al. 1997, 1998), or fish oil (Hammer \& Wills, 1978; Kobatake et al. 1983; Mouri et al. 1984; Hu et al. 1989; Song et al. 2000) enhanced the susceptibility of the liver and kidney to lipid peroxidation, whereas in the brain and testis lipid peroxide levels were not increased in rats with adequate vitamin $\mathrm{E}$ nutritional status (Kubo et al. 1998). Enhancement of lipid peroxidation was a function of the dietary DHA level and also increased the requirement for vitamin $\mathrm{E}$, a lipophilic membrane antioxidant (Saito et al. 1996; Kubo et al. 1998). This is thought to be attributable to the partial substitution of membrane

\footnotetext{
Abbreviations: DHA, docosahexaenoic acid; EPA, eicosapentaenoic acid; GSH, glutathione; LA, linoleic acid; $\alpha$-LN, $\alpha$-linolenic acid; PUFA, polyunsaturated fatty acids; SH, sulfhydryl; TBA, thiobarbituric acid.

* Corresponding author: Dr Morio Saito, fax +8133202 3278, email msaito@nih.go.jp
} 
fatty acids with DHA, which is very susceptible to lipid peroxidation and thus potentially unstable (Kubo et al. 1998).

Nevertheless, in an earlier study, we found that in rats ingesting DHA along with an adequate level of vitamin $\mathrm{E}$, the extent of tissue lipid peroxidation was less than expected from the relative peroxidizability index which was calculated from the fatty acid composition of the total tissue lipids (Kubo et al. 1998, 2000). This phenomenon was especially prominent in the liver and, to a lesser extent, in the testis (Kubo et al. 2000). However, the tissue lipid peroxide levels nearly coincided with the peroxidizability indices for total tissue lipids when a vitamin E-deficient diet was administered (Kubo et al. 2000). In the vitamin E-deficient state, tissue parenchymal cell injuries were also observed.

In the present study, therefore, we fed two other major $n$-3 PUFA, $\alpha$-LN and EPA, to rats to elucidate whether this phenomenon of reduced tissue lipid peroxidation was unique to the administration of highly unsaturated DHA under adequate vitamin $\mathrm{E}$ nutritional status. In addition, we analysed changes in lipid peroxide scavenger levels to clarify the mechanism of the suppression of lipid peroxidation. To differentiate the effect of each n-3 PUFA, we employed purified EPA and DHA as a model, as well as perilla oil as a source of $\alpha-\mathrm{LN}$.

\section{Materials and methods}

\section{Animals and diets}

The experimental procedures used in the present study met the guidelines of the Animal Committee of the Independent Administrative Institution, National Institute of Health and Nutrition (Tokyo, Japan).

Male Sprague-Dawley rats (Japan SLC, Hamamatsu, Japan), 5 weeks of age and weighing 100-133 g, were housed individually in stainless steel wire-bottomed cages at a constant temperature of $22 \pm 1{ }^{\circ} \mathrm{C}$ and relative humidity of $50-60 \%$ with a $12 \mathrm{~h}$ light-dark cycle. The composition of the experimental diets, based on the AIN-76 purified diet for rats (American Institute of Nutrition, 1977, 1980), is shown in Table 1. The lipid content of the diets was $100 \mathrm{~g} / \mathrm{kg}$ diet and $21.6 \%$ of the total energy. EPA ethyl esters (96\% pure) prepared from sardine oil and DHA ethyl esters ( $83 \%$ pure) prepared from orbital fat of tuna were donated by Nippon Suisan Kaisha (Hachioji, Japan) and Maruha Corporation (Tsukuba, Japan), respectively. To prevent the auto-oxidation of DHA, EPA and $\alpha-\mathrm{LN}$ in the diets, they were prepared beforehand without adding these PUFA and stored at $-20^{\circ} \mathrm{C}$. DHA and EPA were stored at $-80^{\circ} \mathrm{C}$ and Perilla oil rich in $\alpha-\mathrm{LN}$ was stored at $-20^{\circ} \mathrm{C}$. They were mixed with each diet every day immediately before feeding. The vitamin E concentrations as RRR- $\alpha$-tocopherol equivalents of the control diet (linoleic acid (LA; $18: 2 n$ 6) group) and the test diets ( $\alpha$-LN, EPA and DHA groups) were $54 \mathrm{mg} / \mathrm{kg}$. The vitamin $\mathrm{E}$ concentration of the DHA diet with low vitamin E (DHA + low vitamin E group) was $7.5 \mathrm{mg} / \mathrm{kg}$. The RRR- $\alpha-$, RRR- $\beta-$, RRR- $\gamma-$ and $\mathrm{RRR}-\delta$-tocopherols biopotency ratio was
$100: 25: 5: 0 \cdot 1$, respectively, in the calculation of RRR$\alpha$-tocopherol equivalent (Mino et al. 1988). All-rac- $\alpha-$ tocopheryl acetate ( $>99 \%$ pure) was used to adjust the vitamin $\mathrm{E}$ concentration in the diets.

The fatty acid composition ( $\mathrm{g} / 100 \mathrm{~g}$ fatty acids) of dietary lipids is also indicated in Table 1. The control lipid (LA group), devoid of EPA and DHA, contained $41 \mathrm{~g}$ $\mathrm{LA} / 100 \mathrm{~g}$, which is comparable to the $\alpha-\mathrm{LN}(39.9 \mathrm{~g} /$ $100 \mathrm{~g})$, EPA $(37 \cdot 8 \mathrm{~g} / 100 \mathrm{~g})$ and DHA $(37 \cdot 2 \mathrm{~g} / 100 \mathrm{~g})$ levels of the test lipids. In addition, all the test lipids $(\alpha-\mathrm{LN}$, EPA, DHA and DHA + low vitamin E groups) were prepared to provide a constant amount of LA as essential $n$-6 PUFA at at least $2 \cdot 1 \%$ of total dietary energy, where the proportion was approximately $10 \%$. The degree of unsaturation of the dietary lipids is presented as the double-bond index (Pietrangelo et al. 1990) and the peroxidizability index (Hu et al. 1989). It has been reported that the relative reaction rate constants of peroxidation were 1 , 2, 3, 4 and 5 against PUFA in which the number of methylene groups among the double bonds was one, two, three, four and five, respectively (Cosgrove et al. 1987). The peroxidizability index of the lipid, therefore, is calculated from its fatty acid composition (\%, w/w) according to the following equation (Hu et al. 1989):

peroxidizability index $=(\%$ dienoic $\times 1)+(\%$ trienoic $\times 2)$

$$
\begin{aligned}
& +(\% \text { tetraenoic } \times 3) \\
& +(\% \text { pentaenoic } \times 4) \\
& +(\% \text { hexaenoic } \times 5)
\end{aligned}
$$

After the rats were fed the basal diet containing $50 \mathrm{~g}$ olive oil/kg diet for $4 \mathrm{~d}$, six or seven rats in each group were fed the experimental diets for $22 \mathrm{~d}$. Food and water were consumed ad libitum. Each diet was made available to the rats in the evening and removed the next morning. After being deprived of food for $9 \mathrm{~h}$, the rats were killed by cardiac puncture. The tissues were promptly excised, washed with isotonic saline and weighed. The liver was perfused with ice-cold isotonic saline via the portal vein. The liver, kidney and testis samples were stored at $-80^{\circ} \mathrm{C}$ until needed for analysis. Serum was separated by centrifugation at $2700 \mathrm{~g}$ for $15 \mathrm{~min}$ at $4^{\circ} \mathrm{C}$.

\section{Conjugate diene and chemiluminescence intensity analyses}

Liver microsomes were prepared (Saito \& Yamaguchi, 1988), and the conjugated dienes of the liver microsomes and tissues were determined by the method of $\mathrm{Hu}$ et al. (1989) and Rao \& Recknagel (1968). The microsomal protein content was measured by the method of Lowry et al. (1951).

The liver, kidney and testis chemiluminescence intensities of the homogenates were determined according to the method of Miyazawa et al. (1984). The light emitted from the homogenates is due mainly to singlet molecular oxygen and/or excited carbonyl compounds resulting from the breakdown of lipid peroxyradicals (Boveris et al. 
Table 1. Composition of experimental diets and fatty acid composition of dietary lipids given to rats $^{\star}$

\begin{tabular}{|c|c|c|c|c|c|}
\hline Diet. .. & LA & $\alpha-\mathrm{LN}$ & EPA & $\mathrm{DHA}$ & $\mathrm{DHA}+$ lowVit E \\
\hline$n-3$ Fatty acids (\% total energy) & - & $8 \cdot 6$ & $8 \cdot 2$ & $8 \cdot 0$ & $8 \cdot 0$ \\
\hline LA (\% total energy) & 8.9 & $2 \cdot 4$ & $2 \cdot 2$ & $2 \cdot 1$ & $2 \cdot 1$ \\
\hline Basic components† (g/kg diet) & $900 \cdot 0$ & $900 \cdot 0$ & $900 \cdot 0$ & $900 \cdot 0$ & $900 \cdot 0$ \\
\hline Test lipids $\ddagger$ (g/kg diet) & $100 \cdot 0$ & $100 \cdot 0$ & $100 \cdot 0$ & $100 \cdot 0$ & $100 \cdot 0$ \\
\hline Olive oil & $50 \cdot 0$ & $36 \cdot 0$ & $48 \cdot 5$ & $37 \cdot 2$ & $37 \cdot 2$ \\
\hline Safflower-seed oil & $50 \cdot 0$ & 0 & $8 \cdot 2$ & $9 \cdot 0$ & $9 \cdot 0$ \\
\hline Perilla oil & 0 & $64 \cdot 0$ & 0 & 0 & 0 \\
\hline EPA concentrate§ & 0 & 0 & $43 \cdot 3$ & 0 & 0 \\
\hline DHA concentrate\| & 0 & 0 & 0 & $53 \cdot 8$ & $53 \cdot 8$ \\
\hline \multicolumn{6}{|l|}{ Fatty acid $(g / 100 g)$} \\
\hline $16: 0$ & $8 \cdot 7$ & $9 \cdot 0$ & $6 \cdot 6$ & $4 \cdot 7$ & $4 \cdot 7$ \\
\hline $16: 1 n-7$ & 0.6 & 0.6 & 0.5 & 0.4 & 0.4 \\
\hline $18: 0$ & $2 \cdot 8$ & $2 \cdot 1$ & $1 \cdot 8$ & 1.5 & 1.5 \\
\hline $18: 1 n-9$ & $45 \cdot 4$ & $36 \cdot 3$ & 40.5 & $31 \cdot 2$ & $31 \cdot 2$ \\
\hline $18: 2 n-6$ LA & $41 \cdot 0$ & $11 \cdot 3$ & $10 \cdot 1$ & $9 \cdot 8$ & $9 \cdot 8$ \\
\hline $18: 3 n-3$ & 0.9 & 39.9 & 0.5 & 0 & 0 \\
\hline $20: 4 n-6$ AA & 0 & 0 & 0.9 & 0.4 & 0.4 \\
\hline $20: 5 n-3$ EPA & 0 & 0 & $37 \cdot 8$ & 1.5 & 1.5 \\
\hline $22: 1 n-9+22: 1 n-11$ & 0 & 0 & 0 & $1 \cdot 8$ & $1 \cdot 8$ \\
\hline $22: 3 n-3$ & 0 & 0 & 0 & 1.4 & 1.4 \\
\hline $22: 5 n-3$ & 0 & 0 & 0 & $3 \cdot 0$ & $3 \cdot 0$ \\
\hline $22: 6 n-3 \mathrm{DHA}$ & 0 & 0 & 0 & $37 \cdot 2$ & $37 \cdot 2$ \\
\hline Others & 0.6 & 0.8 & $1 \cdot 1$ & $7 \cdot 0$ & $7 \cdot 0$ \\
\hline Double-bond index & $1 \cdot 31$ & 1.77 & $2 \cdot 19$ & $3 \cdot 17$ & 3.17 \\
\hline Peroxidizability index ${ }^{\star *}$ & $42 \cdot 8$ & 89.3 & $166 \cdot 2$ & $225 \cdot 9$ & 225.9 \\
\hline
\end{tabular}

LA, linoleic acid; $\alpha$-LN, $\alpha$-linolenic acid; EPA, eicosapentaenoic acid; DHA, docosahexaenoic acid; Vit E vitamin $\mathrm{E} ; \mathrm{AA}$, arachidonic acid.

*The energy density of all the diets was $17.4 \mathrm{MJ} / \mathrm{kg}$ diet $(4160 \mathrm{kcal} / \mathrm{kg})$ using the Atwater energy factors for the energy calculation (Atwater, 1910). The vitamin E content as RRR- $\alpha$-tocopherol equivalent of the LA, $\alpha$-LN, EPA and DHA diets was $54 \mathrm{mg} / \mathrm{kg}$ and that of the DHA + lowVit E diet was $7.5 \mathrm{mg} / \mathrm{kg}$, respectively.

†The basic components of the diet given to all the groups were as follows: casein, $200.0 \mathrm{~g}$; DL-methionine, $3.0 \mathrm{~g}$; maize starch, $150.0 \mathrm{~g}$; sucrose, $225.0 \mathrm{~g}$; glucose, $225.0 \mathrm{~g}$; cellulose powder, $50.0 \mathrm{~g}$; AlN-76 vitamin mixture (America Institute of Nutrition, 1977, 1980), 10.0 g; AlN-76 mineral mixture (America Institute of Nutrition, 1977, 1980), 35.0 g; choline bitartrate, $2.0 \mathrm{~g}$.

† Fat energy percentage was $21.6 \%$ of total energy.

$\S$ EPA ethyl esters prepared from fatty acid ethyl esters of sardine oil were used and the purity was $96 \%$.

$\| \mathrm{DHA}$ ethyl esters prepared from fatty acid ethyl esters of orbital fat of tuna were used and the purity was $83 \%$. Major fatty acids of the remaining $17 \%$ were as follows: $20: 4 n-6,0.7 ; 20: 5 n-3,2.3 ; 22: 1 n-11,1.2$; $22: 1 n-9,0.8 ; 22: 3 n-3,4 \cdot 6 ; 22: 5 n-3,4.0 ;$ others, $3.4 \%$.

I Double-bond index expresses mean double bond number and is the sum of the fraction of each fatty acid multiplied by the number of double bonds in that acid (Pietrangelo et al. 1990).

** Peroxidizability index (Hu et al. 1989) is calculated as follows: peroxidizability index $=(\%$ dienoic $\times$

$1)+(\%$ trienoic $\times 2)+(\%$ tetraenoic $\times 3)+(\%$ pentaenoic $\times 4)+(\%$ hexaenoic $\times 5)$.

1981; Miyazawa et al. 1981), which are produced in the early stage of peroxidation.

\section{Thiobarbituric acid value and fluorescent substance analyses}

The serum thiobarbituric acid (TBA) value was determined by the method of Yagi (1976). Butylated hydroxytoluene (BHT) as an antioxidant was added to the reaction mixture at a final concentration of $0.36 \mathrm{mmol} / \mathrm{l}$. The tissue TBA values were measured according to the method of Ohkawa et al. (1979) with a minor modification, in which BHT was added to the reaction mixture at a final concentration of $0.45 \mathrm{mmol} / \mathrm{l}$. TBA values are expressed in terms of the malondialdehyde equivalent.

Serum water-soluble fluorescent substances were analysed by the method of Tsuchida et al. (1985). Liver microsomal lipofuscin concentration was determined by the method of Fletcher et al. (1973).
Vitamin E, total ascorbic acid and non-protein sulfhydryl assays

The vitamin E levels in the test lipids, serum and tissues were analysed by HPLC as previously described (Saito et al. 1992).

Total ascorbic acid (Roe et al. 1948) and non-protein sulfhydryl (SH) (Beutler et al. 1963) levels in the tissues were measured. The non-protein $\mathrm{SH}$ component consists mostly of glutathione (GSH).

\section{Selenium-dependent glutathione peroxidase assay}

Se-dependent glutathione peroxidase (EC 1.11.1.9) activity was determined according to the method of Noguchi et al. (1973) with a minor modification as previously described (Saito, 1990). 


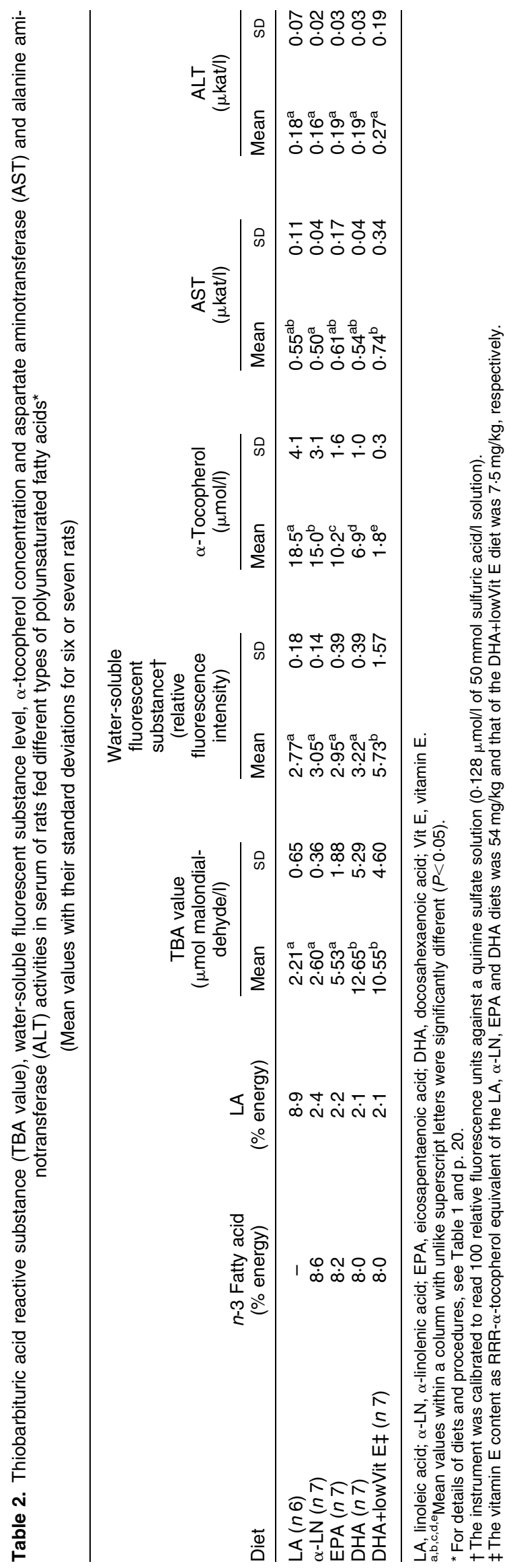

\section{Glutathione reductase and glucose-6-phosphate dehydrogenase assays}

The activities of glutathione reductase $(E C$ 1.6.4.2) and glucose-6-phosphate dehydrogenase $(E C 1 \cdot 1 \cdot 1.49)$ in the liver $12000 \mathrm{~g}$ supernatant fraction were analysed by the methods of Goldberg \& Spooner (1983) and Bergmeyer et al. (1983), respectively.

\section{Serum aspartate aminotransferase and alanine aminotransferase assays}

The activities of aspartate aminotransferase (EC 2.6.1.1) and alanine aminotransferase $(E C 2 \cdot 6 \cdot 1 \cdot 2)$ in the serum were determined with a clinical enzyme assay kit (Wako Pure Chemical, Osaka, Japan) by the method of Reitman \& Frankel (1957).

\section{Fatty acid analysis}

Tissue total lipids were extracted from each tissue according to the method of Folch et al. (1957). The fatty acid compositions of the dietary lipids and total tissue lipids were analysed by GLC with dual-flame ionization detectors (Hitachi 263-30 gas-liquid chromatograph; Tokyo, Japan) by using a $50 \mathrm{~m} \times 0.25 \mathrm{~mm}$ internal diameter glass capillary column coated with CP-Sil-88 (Saito et al. 1990; Kubo et al. 2000). The column temperature was programmed from $160^{\circ} \mathrm{C}$ to $210^{\circ} \mathrm{C}$. $\mathrm{N}_{2}$ was employed as the carrier gas.

\section{Statistical analysis}

After confirming the normality of data and the homogeneity of variance of data for the treatment groups (the latter being evaluated by the Bartlett test), the significance of differences between mean values was assessed by ANOVA coupled with Duncan's multiple-range test at the 1 or $5 \%$ level of significance (Duncan, 1957).

\section{Results}

The rats consumed $14 \cdot 3-14.8 \mathrm{~g}$ food/d and gained 5.4$5.9 \mathrm{~g} / \mathrm{d}$ over the $22 \mathrm{~d}$ experiment. There were no significant differences in food intake and body-weight gain between any of the treatment groups. The weights of the liver, kidney, and testis in the treatment groups changed within small ranges, and the differences were not significant (data not shown).

Serum TBA values were significantly higher in the two DHA groups fed diets with 7.5 or $54 \mathrm{mg}$ vitamin E/kg than in the LA (control), $\alpha-\mathrm{LN}$, and EPA groups (Table 2), but the value in the $\mathrm{DHA}+$ low vitamin $\mathrm{E}$ group was no higher than in the DHA group. The watersoluble fluorescent substance level of the DHA + low vitamin E group was significantly higher than those of the other groups. Serum $\alpha$-tocopherol concentrations were significantly lower in all of the $n-3$ PUFA groups than in the control LA group, and became lower with increasing degrees of unsaturation of dietary PUFA. Serum aspartate aminotransferase activity tended to be higher in the 
Table 3. Conjugated diene level, chemiluminescence intensity, thiobarbituric acid reactive substance (TBA value) and microsomal lipofuscin level in the liver, kidney and testis of rats fed different types of polyunsaturated fatty acids ${ }^{*}$

(Mean values with their standard deviations for six or seven rats)

\begin{tabular}{|c|c|c|c|c|c|c|c|c|c|c|}
\hline \multirow[t]{2}{*}{ Diet. . } & \multicolumn{2}{|c|}{$\mathrm{LA}(n 6)$} & \multicolumn{2}{|c|}{$\alpha-\operatorname{LN}(n 7)$} & \multicolumn{2}{|c|}{$\mathrm{EPA}(n 7)$} & \multicolumn{2}{|c|}{$\mathrm{DHA}(n 7)$} & \multicolumn{2}{|c|}{$\mathrm{DHA}+$ lowVit E† $(n 7)$} \\
\hline & Mean & SD & Mean & SD & Mean & SD & Mean & SD & Mean & SD \\
\hline$n-3$ Fatty acids (\% energy) & - & & $8 \cdot 6$ & & $8 \cdot 2$ & & $8 \cdot 0$ & & $8 \cdot 0$ & \\
\hline LA (\% energy) & 8.9 & & $2 \cdot 4$ & & $2 \cdot 2$ & & $2 \cdot 1$ & & $2 \cdot 1$ & \\
\hline \multicolumn{11}{|l|}{ Liver } \\
\hline $\begin{array}{l}\text { Conjugated diene } \\
(\mu \mathrm{mol} / \mathrm{g})\end{array}$ & $0.97^{\mathrm{a}}$ & 0.09 & $0.95^{a}$ & 0.06 & $1 \cdot 01^{\mathrm{a}}$ & $0 \cdot 13$ & $1 \cdot 24^{\mathrm{b}}$ & $0 \cdot 10$ & $1 \cdot 31^{\mathrm{b}}$ & $0 \cdot 16$ \\
\hline $\begin{array}{l}\text { Microsomal conjugated diene } \\
(\mathrm{nmol} / \mathrm{mg} \text { protein) }\end{array}$ & $13 \cdot 2^{\mathrm{a}}$ & $2 \cdot 2$ & $13 \cdot 9^{\mathrm{ab}}$ & $1 \cdot 7$ & $13 \cdot 8^{\mathrm{ab}}$ & $2 \cdot 1$ & $15 \cdot 9^{b}$ & $1 \cdot 1$ & $15 \cdot 0^{\mathrm{ab}}$ & $2 \cdot 1$ \\
\hline $\begin{array}{l}\text { Chemiluminescence intensity } \\
\text { (count/30 s) }\end{array}$ & $80 \cdot 1^{\mathrm{a}}$ & $34 \cdot 1$ & $89 \cdot 3^{\mathrm{a}}$ & $41 \cdot 7$ & $104 \cdot 2^{\mathrm{a}}$ & $77 \cdot 6$ & $110 \cdot 6^{\mathrm{ab}}$ & $48 \cdot 7$ & $181 \cdot 4^{\mathrm{b}}$ & $98 \cdot 5$ \\
\hline $\begin{array}{l}\text { TBA value } \\
\text { (nmol malondialdehyde/g) }\end{array}$ & $124 \cdot 8^{\mathrm{a}}$ & $16 \cdot 5$ & $149 \cdot 9^{b}$ & $13 \cdot 9$ & $149 \cdot 2^{b}$ & $8 \cdot 2$ & $193 \cdot 6^{c}$ & $23 \cdot 6$ & $209 \cdot 2^{\mathrm{C}}$ & $21 \cdot 7$ \\
\hline $\begin{array}{l}\text { Microsomal lipofuscin } \ddagger \\
\text { (ng/mg protein) }\end{array}$ & $2 \cdot 8^{\mathrm{a}}$ & 0.4 & $2 \cdot 7^{\mathrm{a}}$ & 0.3 & $2 \cdot 6^{\mathrm{a}}$ & 0.3 & $2 \cdot 9^{\mathrm{a}}$ & 0.4 & $2 \cdot 6^{\mathrm{a}}$ & 0.4 \\
\hline \multicolumn{11}{|l|}{ Kidney } \\
\hline $\begin{array}{l}\text { Conjugated diene } \\
(\mu \mathrm{mol} / \mathrm{g})\end{array}$ & $0.67^{\mathrm{a}}$ & 0.11 & $0.64^{a}$ & 0.07 & $0.69^{a b}$ & 0.06 & $0.78^{\mathrm{b}}$ & 0.06 & $0.72^{a b}$ & 0.09 \\
\hline $\begin{array}{l}\text { Chemiluminescence intensity } \\
\text { (count/30 s) }\end{array}$ & $64 \cdot 2^{\mathrm{a}}$ & $28 \cdot 1$ & $73 \cdot 2^{\mathrm{a}}$ & $73 \cdot 7$ & $93 \cdot 3^{\mathrm{a}}$ & $119 \cdot 1$ & $115 \cdot 1^{\mathrm{a}}$ & $125 \cdot 9$ & $167 \cdot 1^{\mathrm{a}}$ & $149 \cdot 0$ \\
\hline $\begin{array}{l}\text { TBA value } \\
\text { (nmol malondialdehyde/g) }\end{array}$ & $132 \cdot 3^{a}$ & 4.0 & $125 \cdot 8^{\mathrm{a}}$ & $9 \cdot 8$ & $129 \cdot 5^{\mathrm{a}}$ & 14.4 & $148 \cdot 2^{b}$ & 4.5 & $157 \cdot 5^{\mathrm{b}}$ & $7 \cdot 3$ \\
\hline \multicolumn{11}{|l|}{ Testis } \\
\hline $\begin{array}{l}\text { Conjugated diene } \\
(\mu \mathrm{mol} / \mathrm{g})\end{array}$ & $0 \cdot 21^{a}$ & 0.03 & $0.23^{\mathrm{ab}}$ & 0.05 & $0.22^{a}$ & 0.03 & $0.27^{b}$ & 0.01 & $0.24^{\mathrm{ab}}$ & 0.03 \\
\hline $\begin{array}{l}\text { Chemiluminescence intensity } \\
\text { (count/30 s) }\end{array}$ & $52 \cdot 0^{\mathrm{a}}$ & $19 \cdot 5$ & $47 \cdot 4^{\mathrm{a}}$ & $26 \cdot 0$ & $40 \cdot 6^{a}$ & $15 \cdot 5$ & $35 \cdot 7^{\mathrm{a}}$ & 9.7 & $44.0^{\mathrm{a}}$ & $12 \cdot 0$ \\
\hline $\begin{array}{l}\text { TBA value } \\
\text { (nmol malondialdehyde/g) }\end{array}$ & $29 \cdot 5^{\mathrm{ab}}$ & $1 \cdot 6$ & $27 \cdot 1^{\mathrm{a}}$ & $2 \cdot 5$ & $25 \cdot 0^{\mathrm{a}}$ & 2.5 & $24.9^{a}$ & 1.8 & $32.9^{b}$ & 9.5 \\
\hline
\end{tabular}


DHA + low vitamin E group than in the control LA group, but the difference was not significant (Table 2). A similar tendency was also observed for alanine aminotransferase activity.

Conjugated diene levels in liver homogenates were significantly higher in the two DHA-fed groups than in the other groups, but the value in the DHA + low vitamin E group was no higher than that in the DHA group (Table 3). A similar trend was also observed in liver microsomal conjugated diene concentration. Liver chemiluminescence intensity was significantly greater than in the control only in the DHA + low vitamin E group. Liver TBA values were significantly higher in all $n$-3 PUFA groups than in the control group, and values in the two DHA-fed groups were greater than in the $\alpha$-LN and EPA groups, although there was no significant difference between the two DHA groups. Liver microsomal lipofuscin concentration did not differ among any of the treatment groups.

The conjugated diene level in the kidney homogenates was significantly higher in the DHA group than in the control LA group, but there was no significant difference among the other LA, $\alpha$-LN, EPA, and DHA + low vitamin E groups (Table 3). The chemiluminescence intensities tended to be higher in the two DHA groups, but no significant difference was noticed in the values among any of the treatment groups, owing to considerable scattering of the values. The kidney TBA values in the two DHA groups were significantly greater than in the control group, but the value in the DHA + low vitamin E group was no higher than in the DHA group.
The conjugated diene level in the testis homogenates was significantly higher in the DHA group than in the control LA group (Table 3). No significant difference was recognized in chemiluminescence intensity among any of the treatment groups. The TBA value was significantly higher in the DHA + low vitamin E group than in the other treatment groups, except for the control.

Liver $\alpha$-tocopherol levels showed a similar trend to serum level (Table 4). Liver ascorbic acid levels were significantly higher in all the $n-3$ PUFA groups than in the control LA group, but there was no significant difference among the $n-3$ PUFA groups. A similar trend was observed in non-protein SH concentrations, with the exception of the DHA + low vitamin E group, which showed no significant difference from the control LA group. Glutathione peroxidase activity in the two DHA groups was significantly lower than in the control, irrespective of the fact that the tissue lipid peroxide levels were higher in the two DHA groups. Glutathione reductase activity in the treatment groups changed within a small range, and the differences were insignificant. Glucose-6-phosphate dehydrogenase activity was significantly lower in all of the $n-3$ PUFA groups than in the control LA group, and became lower with increasing degrees of unsaturation of dietary PUFA.

Kidney $\alpha$-tocopherol levels in the two DHA groups were significantly lower than in the control, but those of the $\alpha-\mathrm{LN}$ and EPA groups did not decrease (Table 4). The ascorbic acid concentration was significantly higher or tended to be higher in all of the $n-3$ PUFA groups compared with the control. Non-protein SH levels were significantly higher than in the control only in the two DHA

Table 4. Lipid peroxide scavengers in the liver, kidney and testis of rats fed different types of polyunsaturated fatty acids* (Mean values with their standard deviations for six or seven rats)

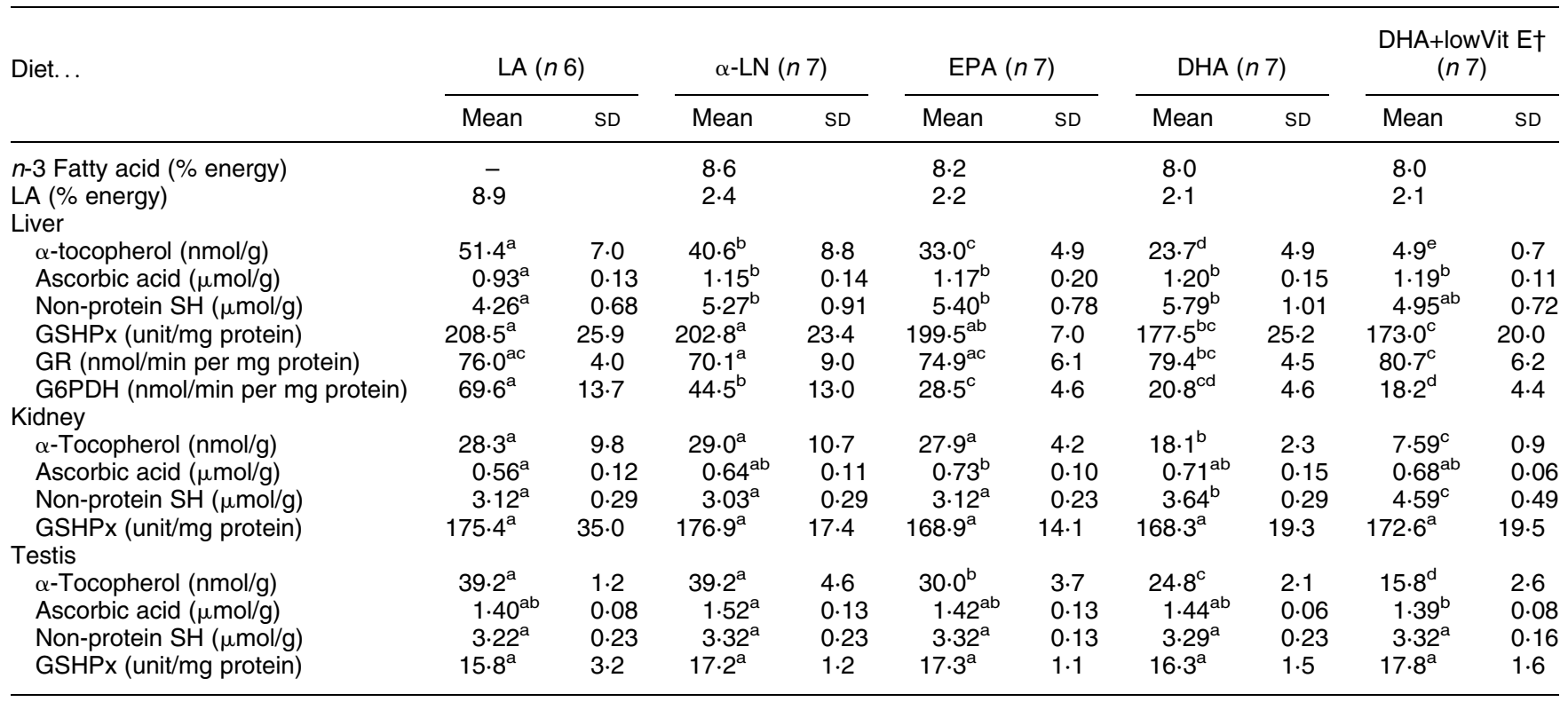

LA, linoleic acid; $\alpha$-LN, $\alpha$-linolenic acid; EPA, eicosapentaenoic acid; DHA, docosahexaenoic acid; Vit E, vitamin E; SH, sulfhydryl; GSHPx, glutathione peroxidase; GR, glutathione reductase; G6PDH, glucose-6-phosphate dehydrogenase.

a,b,c,d,e Mean values within a row with unlike superscript letters were significantly different $(P<0.05)$

${ }^{*}$ For details of diets and procedure, see Table 1 and p. 20.

†The vitamin E content as RRR- $\alpha$-tocopherol equivalent of the LA, $\alpha$-LN, EPA and DHA diets was $54 \mathrm{mg} / \mathrm{kg}$ and that of the DHA+lowVit E diet was $7.5 \mathrm{mg} / \mathrm{kg}$, respectively. 
groups; the level in the DHA + low vitamin E group was significantly greater than that in the DHA group. No significant difference in glutathione peroxidase activity was found among any of the treatment groups.

Testis $\alpha$-tocopherol levels of the EPA and DHA groups were significantly lower than that of the control, and the level in the DHA group was significantly lower than that of the EPA group (Table 4). Ascorbic acid and non-protein SH concentrations and glutathione peroxidase activity did not change significantly.

The liver peroxidizability indices were 85.3 (SD 9.6), 95.0 (SD 14.4), 139.3 (SD 5.3), and 160.6 (SD 9.3) for the control LA, $\alpha$-LN, EPA and DHA groups, respectively. The control LA group peroxidizability index was significantly different $(P<0.01)$ from those of the EPA and DHA groups, that of the $\alpha$-LN group was also significantly different $(P<0.01)$ from the EPA and DHA groups, that of the EPA group was significantly different to all other groups $(P<0 \cdot 01)$, and that of the DHA group was also significantly different to all other groups $(P<0 \cdot 01)$. As no significant influence of dietary vitamin $\mathrm{E}$ levels on the fatty acid composition of the liver was reported (Kaasgaard et al. 1992; Kubo et al. 2000), a pooled sample from seven rats in the DHA + low vitamin E group was analysed to calculate the peroxidizability index, which was $152 \cdot 1$. Because there were inadequate amounts of tissue in the kidney and testis samples, the fatty acid compositions of these organs were analysed by using pooled samples from six rats from the LA group and seven rats each from the $\alpha$-LN, EPA, DHA, and DHA + low vitamin E groups. The kidney peroxidizability indices were $98 \cdot 8$, $110.1,129.9,132.9$ and 128.6 for the LA, $\alpha-\mathrm{LN}$, EPA, DHA, and DHA + low vitamin E groups, respectively. Those of the testis were $113.2,111.2,119.2,118.7$ and 122.9 for the same groups, respectively.

Fig. 1 shows the relationship between peroxidizability indices and lipid peroxide levels in the liver, kidney, and testis, expressed relative to the control values. In the liver, the relative peroxidizability indices of total lipids increased as the degrees of unsaturation of dietary PUFA increased (Fig. 1 (A)). The relative chemiluminescence intensity for the DHA + low vitamin E group was 2.3 times the control value, and higher than the relative peroxidizability index. The relative TBA value for the same group coincided with the relative peroxidizability index. On the other hand, the relative values of lipid peroxides, as assessed by levels of conjugated diene, chemiluminescence emission, and TBA-reactive substances in the EPA and DHA groups, were much lower than the respective relative peroxidizability index. However, the relative values of lipid peroxides in the $\alpha$-LN group were closely associated with the peroxidizability index.

In the kidney, the relative peroxidizability indices of the total lipids increased as the degrees of unsaturation of dietary PUFA increased, but to a lesser extent than in the liver (Fig. 1 (B)). The relative values of lipid peroxides in the $\alpha$-LN group seemed to be associated with the relative peroxidizability index. The relative values of lipid peroxides, except for the chemiluminescence intensities, in the EPA and DHA groups appeared to be lower than the respective

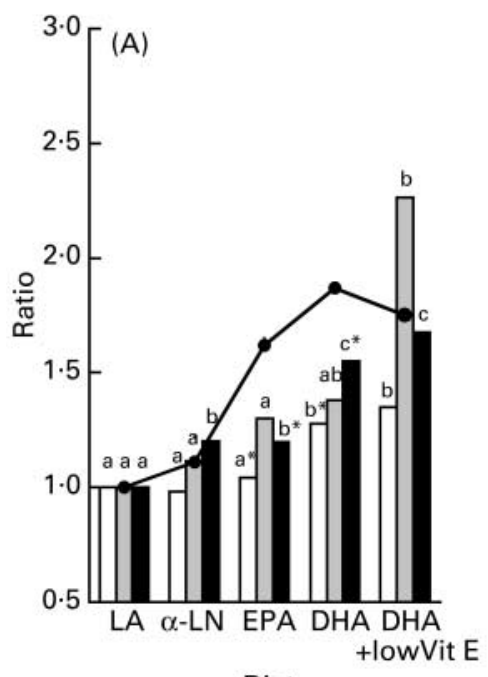

Diet

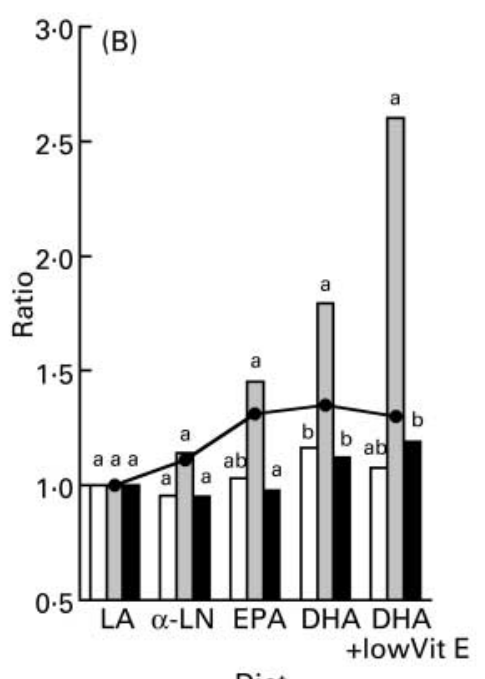

Diet

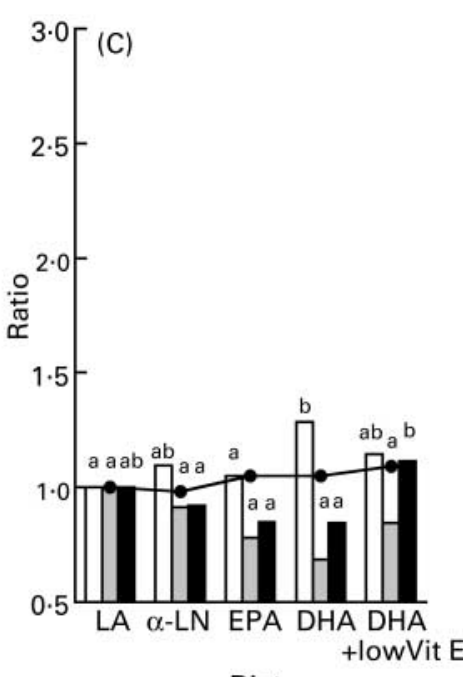

Diet

Fig. 1. Lipid peroxide levels $(\square, \square, \square)$ and peroxidizability indices of the total lipids $(-\bigcirc-)$ expressed relative (as a ratio) to the control values (linoleic acid (LA) group) in the liver (A), kidney (B) and testis $(C)$ of rats fed different $n$ - 3 polyunsaturated fatty acids. The peroxidizability index was used in the present study as an indicator to determine the vulnerability of tissue lipids to oxidation. The peroxidizability index of lipids was calculated according to the following equation: peroxidizability index $=($ dienoic $\% \times 1)+($ trienoic $\% \times 2)+($ tetraenoic $\% \times 3)+($ pentaenoic $\% \times 4)+($ hexaenoic $\% \times 5$ ). ( $\square$ ), Conjugated diene levels; $(\square)$, chemiluminescence intensities; ( $\square$ ), thiobarbituric acid values. Mean values are shown for six rats fed the LA diet and for seven rats fed the other $n-3$ polyunsaturated fatty acids diets. ${ }^{a, b, c}$ Mean values for the same analytical item in each tissue with unlike letters were significantly different by ANOVA coupled with the Duncan's multiple-range test $(P<0.05)$. *Significant difference by Student's $t$ test between the relative increase of peroxidizability index and that of each lipid peroxide in the liver except for the docosahexaenoic acid (DHA) + low vitamin $\mathrm{E}$ (Vit $\mathrm{E})$ group $(P<0.01)$. The fatty acid analysis to calculate the peroxidizability index of the DHA + lowVit E group was carried out using a pooled sample from seven rats. The fatty acid analyses for the kidney and testis were also conducted using pooled samples from six rats of the LA group and from seven rats of the $\alpha$-linolenic acid ( $\alpha$-LN), eicosapentaenoic acid (EPA), DHA and DHA + lowVit E groups. 
relative peroxidizability indices. The relative TBA value of the DHA + low vitamin E group in the kidney seemed to be correlated well with the relative peroxidizability index. The chemiluminescence intensities in the two DHA groups were higher than their relative peroxidizability indices, although the relative intensities were not significantly different among any of the treatment groups.

In the testis, the relative peroxidizability indices of the total lipids in the $n-3$ PUFA groups were 1.0 to 1.1 times the control value (Fig. 1 (C)). The relative conjugated diene levels in the treatment groups, except for the DHA group, seemed to be associated with each relative peroxidizability index. The TBA value for the DHA + low vitamin $\mathrm{E}$ group and the chemiluminescence intensity and TBA value for the $\alpha$-LN group also appeared to coincide with their relative peroxidizability indices. The relative chemiluminescence intensities and TBA values for the EPA and DHA groups were 0.7 to 0.85 times the control values, and the levels seemed to be lower than their relative peroxidizability indices.

\section{Discussion}

The potentially harmful influences of fish oils and fish-oil products rich in EPA and DHA have been overlooked because of the health benefits conferred by $n-3$ PUFA. Therefore, we considered that a study to clarify the mechanisms suppressing dietary $n$-3 PUFA-induced lipid peroxidation below the relative peroxidizability index was important for reasons of safety, and also to define a means of efficiently enhancing the physiological efficacy of $n-3$ PUFA.

We chose in the present study three typical tissues and dietary n-3 PUFA; that is, liver, kidney, and testis, and $\alpha-\mathrm{LN}, \mathrm{EPA}$, and DHA, for the reasons described earlier (p. 000). The peroxidizability indices ratio of the dietary lipids was approximately 1:2:4:5 for the control LA, $\alpha$-LN, EPA and DHA diets, respectively (Table 1 ).

Our results show that serum lipid peroxidation, as assessed by the level of TBA-reactive substances, and the antioxidant $\alpha$-tocopherol were correlated inversely with increasing degrees of unsaturation of dietary PUFA (Table 2). Additionally, under a very low supply of vitamin $\mathrm{E}$ (DHA + low vitamin E group), the water-soluble fluorescent substance level and aspartate aminotransferase and alanine aminotransferase activities were higher than those of the control, indicating the potential for tissue parenchymal cell injuries in rats given as little as $7.5 \mathrm{mg}$ vitamin E/ $\mathrm{kg}$ diet. In this vitamin E-deficient nutritional state, tissue parenchymal cell injuries are thought to be promoted through enhanced lipid peroxidation, and thus the endproducts of lipid peroxidation, i.e. water-soluble fluorescent substances, appear in the blood. Farwer et al. (1994) reported that when fish oil was administered to rats, yellow fat was recognized in the storage fat in which lipofuscin pigments were accumulated, especially with an insufficient supply of dietary vitamin E. Hence, we thought that lipid peroxidation was not stimulated to induce tissue parenchymal cell injuries under adequate vitamin E nutritional status, even after large amounts of highly unsaturated DHA were given to the rats.
In the liver, lipid peroxidation, as assessed by the conjugated diene, chemiluminescence emission and TBA-reactive substances, together with the relative peroxidizability indices, became higher (Table 3, Fig. 1 (A)), and $\alpha$-tocopherol levels inversely lower (Table 4) with increasing degrees of unsaturation of dietary PUFA. When a deficient level of vitamin E was supplied (DHA + low vitamin E group), levels of relative TBA-reactive substances nearly coincided with the relative peroxidizability index. However, the relative chemiluminescence intensity for the DHA + low vitamin E group was higher than the relative peroxidizability index. In chemiluminescence analysis, light is emitted mainly from singlet molecular oxygen and/or excited carbonyl compounds yielded by the breakdown of lipid peroxyradicals (Boveris et al. 1981; Miyazawa et al. 1981). With low dietary vitamin E, which approximates to vitamin E deficiency, the chain reaction of lipid peroxidation to form peroxyradicals of fatty acids such as highly unsaturated DHA may not be suppressed easily. In the lipid peroxides analysed, TBAreactive substances which were formed in a relatively late stage of lipid peroxidation compared with the earlystage products such as conjugated diene and chemiluminescence emission, reflected most of the respective dietary $n-3$ PUFA-induced lipid peroxidation. Similar results were also observed in vitro by Visioli et al. (1998). We noticed a close association between the relative peroxidizability index and lipid peroxidation in the $\alpha$-LN group (Fig. 1 (A)). However, the extent of lipid peroxidation in the EPA and DHA groups was far less than expected from their relative peroxidizability indices. Therefore, this phenomenon is not unique to DHA, which has six double bonds, but also occurs with EPA, which has five double bonds, in the liver under adequate vitamin $\mathrm{E}$ nutritional status.

In the kidney, the changes and relationships between the relative peroxidizability indices for the total lipids and the extent of lipid peroxidation were similar to those observed in the liver, i.e. both the indices and the lipid peroxidation increased as the degrees of unsaturation of dietary PUFA increased, but to a lesser extent than in the liver, even in the EPA and DHA groups (Fig. 1 (B)).

In the testis, almost no increase was observed in the relative peroxidizability indices for total lipids, even after a high intake of DHA or EPA (Fig. 1 (C)). Therefore, the extent of lipid peroxidation also did not increase, on the whole, in the testis, irrespective of the degree of unsaturation of dietary PUFA and the vitamin E level (Table 3, Fig. 1 (C)). Accordingly, the susceptibility to lipid peroxidation is lower in the testis than in the liver and kidney, as was observed previously (Kubo et al. 1998, 2000).

Our results show a characteristic pattern of changes, in both the extent of lipid peroxidation and the relative peroxidizability indices in each tissue. Accordingly, to clarify the mechanisms of the suppression of lipid peroxidation, variations in the lipid peroxide scavengers were further investigated.

The $\alpha$-tocopherol concentration in the liver decreased remarkably with increasing degrees of unsaturation of dietary PUFA, but the levels of both ascorbic acid and GSH increased significantly in all of the $n$-3 PUFA groups, 
probably to compensate for the decrease in $\alpha$-tocopherol (Table 4). It has been suggested that three antioxidants, vitamin E, ascorbic acid, and GSH, are interrelated in the maintenance of tissue vitamin E levels. Reductive recycling by ascorbic acid of $\alpha$-tocopheroxyl radicals is known to occur (Tappel, 1962; Meister, 1992; Wells et al. 1995), and the enzymic and non-enzymic reductive regeneration of ascorbic acid from oxidized ascorbic acid by GSH is also known (Winkler et al. 1994). Thus the antioxidant efficacy of $\alpha$-tocopherol is potentiated by the action of ascorbic acid and GSH. The activity of glutathione reductase in reproducing ascorbic acid from oxidized ascorbic acid via the GSH-dependent system in the liver did not change significantly, and that of glucose-6-phosphate dehydrogenase in supplying NADPH for the GSHdependent system decreased with increasing degrees of unsaturation of dietary PUFA (Table 4). Hence, the biosynthesis of ascorbic acid and GSH, not the reproduction of reduced forms of ascorbic acid and GSH from their oxidized forms, may be promoted as the lipid peroxidationinduced increase in the requirement for vitamin $\mathrm{E}$ is enhanced in the liver. This probable potentiation of the antioxidant system may explain, at least in part, the observation that the extent of lipid peroxidation in the EPA and DHA groups fed diets with adequate level of vitamin $\mathrm{E}$ was far less than expected from the relative peroxidizability indices of the total liver lipids.

In the kidney, the levels of lipid peroxide scavengers were lower, on the whole, than those of the liver and testis (Table 4). However, the increase in the relative peroxidizability index of total lipids was also moderate or low, even after highly unsaturated DHA was given to the animals (Fig. 1 (B)). Accordingly, in the kidney the extent of lipid peroxidation may be closer to the relative peroxidizability indices, even in the EPA and DHA groups, than in the liver.

The testis was insensitive to lipid peroxidation under adequate vitamin E nutritional status, even after a high intake of highly unsaturated DHA or EPA (Fig. 1 (C)). Presumably this phenomenon was ascribable primarily to the negligible increase in the relative peroxidizability index and to the higher levels of $\alpha$-tocopherol than those of other tissues, even in the low vitamin E $(7.5 \mathrm{mg} / \mathrm{kg})$ group (Table 4). Moreover, the ascorbic acid level of the testis was retained at a higher level (about 2.5 times that of the kidney) (Table 4), suggesting effective $\alpha$-tocopherol recycling mediated by ascorbic acid and the GSH-dependent system. Therefore, the testis probably contains enough lipid peroxide scavengers to suppress lipid peroxidation, even after a high DHA or EPA intake.

Because DHA and EPA are the major PUFA in fish oils, the phenomena seen here with DHA and EPA intakes may also be applicable to fish oil and fish-oil supplement intakes.

In conclusion, our results suggest that antioxidative suppression of lipid peroxidation below the relative peroxidizability index is not unique to DHA, which has six double bonds, but also occurs with EPA, which has five double bonds, under adequate vitamin $\mathrm{E}$ nutritional status. However, it does not occur with $\alpha-\mathrm{LN}$, which has three double bonds.

\section{Acknowledgements}

We gratefully acknowledge the technical assistance of $\mathrm{Mr}$ Hidenori Otokita. We thank Maruha Corporation of Japan and Nippon Suisan Kaisha, Ltd. for the generous donation of DHA and EPA, respectively. This work was supported by a Health Science Research Grant from the Ministry of Health, Labour and Welfare, by a Grant-in-Aid for Scientific Research from the Ministry of Education, Culture, Science, Sports and Technology, and by the Skylark Food Science Institute Academic Research Grant Program, Japan.

\section{References}

American Institute of Nutrition (1977) Report of the American Institute of Nutrition Ad Hoc Committee on Standards for Nutritional Studies. Journal of Nutrition 107, 1340-1348.

American Institute of Nutrition (1980) Second report of the Ad Hoc Committee on Standards for Nutritional Studies. Journal of Nutrition 110, 1726.

Atwater WO (1910)??? Principles of Nutrition and Nutritive Value of Food. US Department of Agriculture Farmers' Bulletin no. 142, 2nd revision, p. 48.

Bergmeyer HU, Graßl M \& Walter H-E (1983) Glucose-6-phosphate dehydrogenase from yeast. In Methods of Enzymatic Analysis, 3rd ed. vol. II, pp. 202-203 [HU Bergmeyer, J Bergmeyer and M Graßl, editors]. Weinheim, Germany: Verlag Chemie GmbH.

Beutler E, Duron O \& Kelly BM (1963) Improved method for the determination of blood glutathione. Journal of Laboratory and Clinical Medicine 61, 882-888.

Boveris A, Cadenas E \& Chance B (1981) Ultraweak chemiluminescence: a sensitive assay for oxidative radical reaction. FASEB Journal 40, 195-198.

Cosgrove JP, Church DF \& Pryor WA (1987) The kinetics of the autoxidation of polyunsaturated fatty acids. Lipids 22, 299-304.

Crawford M, Galli C, Visioli F, Renaud S, Simopoulos AP \& Spector AA (2000) Role of plant-derived omega-3 fatty acids in human nutrition. Annals of Nutrition and Metabolism 44, $263-265$.

Drevon CA (1992) Marine oils and their effects. Nutrition Reviews 50, 38-45.

Duncan DB (1957) Multiple range tests for correlated and heteroscedastic means. Biometrics 13, 164-176.

Dyerberg J (1986) Linolenate-derived polyunsaturated fatty acids and prevention of atherosclerosis. Nutrition Reviews 44, $125-134$.

Farwer SR, Boer BCJD, Haddeman E, Kivits GAA, Wiersma A \& Danse BHJC (1994) The vitamin E nutritional status of rats fed on diets high in fish oil, linseed oil or sunflower seed oil. British Journal of Nutrition 72, 127-145.

Fletcher BL, Dillard CJ \& Tappel AL (1973) Measurement of fluorescent lipid peroxidation products in biological systems and tissues. Analytical Biochemistry 52, 1-9.

Folch J, Lees M \& Sloane-Stanley GA (1957) A simple method for the isolation and purification of total lipides from animal tissues. Journal of Biological Chemistry 226, 497-507.

Goldberg DM \& Spooner RJ (1983) Glutathione reductase. In Methods of Enzymatic Analysis, 3rd ed. vol. III, pp. 258-265 [HU Bergmeyer, J Bergmeyer and M Graßl, editors]. Verlag Chemie GmbH: Weinheim, Germany.

Hammer CT \& Wills ED (1978) The role of lipid components of the diet in the regulation of the fatty acid composition of the rat 
liver endoplasmic reticulum and lipid peroxidation. Biochemical Journal 174, 585-593.

Harris WS (1989) Fish oils and plasma lipid and lipoprotein metabolism in humans: a critical review. Journal of Lipid Research 30, 785-807.

Herold PM \& Kinsella JE (1986) Fish oil consumption and decreased risk of cardiovascular disease: a comparison of findings from animal and human feeding trials. American Journal of Clinical Nutrition 43, 566-598.

Hu M-L, Frankel EN, Leibovitz BE \& Tappel AL (1989) Effect of dietary lipids and vitamin $\mathrm{E}$ on in vitro lipid peroxidation in rat liver and kidney homogenates. Journal of Nutrition 119, $1574-1582$.

Kaasgaard SG, Holmer G, Hoy CE, Behrens WA \& Beare-Rogers JL (1992) Effect of dietary linseed oil and marine oil on lipid peroxidation in monkey liver in vivo and in vitro. Lipids $\mathbf{2 7}$, 740-745.

Kobatake Y, Hirahara F, Innami S \& Nishide E (1983) Dietary effect of n-3 type polyunsaturated fatty acids on serum and liver lipid levels in rats. Journal of Nutritional Science and Vitaminology 29, 11-21.

Kubo K, Saito M, Tadokoro T \& Maekawa A (1997) Changes in susceptibility of tissues to lipid peroxidation after ingestion of various levels of docosahexaenoic acid and vitamin E. British Journal of Nutrition 78, 655-669.

Kubo K, Saito M, Tadokoro T \& Maekawa A (1998) Dietary docosahexaenoic acid does not promote lipid peroxidation in rat tissue to the extent expected from peroxidizability index of the lipid. Bioscience, Biotechnology, and Biochemistry 62, $1698-1706$.

Kubo K, Saito M, Tadokoro T \& Maekawa A (2000) Preferential incorporation of docosahexaenoic acid into nonphosphorus lipids and phosphatidylethanolamine protects rats from dietary DHA-stimulated lipid peroxidation. Journal of Nutrition 130, 1749-1759.

Lands WEM (1992) Biochemistry and Physiology of n-3 fatty acids. FASEB Journal 6, 2530-2536.

Lowry OH, Rosebrough NJ, Farr AL \& Randall RJ (1951) Protein measurement with the Folin phenol reagent. Journal of Biological Chemistry 193, 265-275.

Meister A (1992) On the antioxidant effects of ascorbic acid and glutathione. Biochemical Pharmacology 44, 1905-1915.

Mino M, Tamai H, Yasuda C, Igarashi O, Hayashi M, Hirahara F, Katsui G \& Kijima S (1988) Biopotencies of tocopherol analogues as determined by dialuric acid-induced hemolysis in rats. Vitamins, Japan 62, 241-246.

Miyazawa T, Kaneda T, Takyu C, Yamaguchi A \& Inaba H (1981) Generation of singlet molecular oxygen in rat liver homogenate on adding autoxidized linseed oil. Agricultural and Biological Chemistry 45, 1597-1601.

Miyazawa T, Tsuchida K \& Kaneda T (1984) Riboflavin tetrabutyrate: an antioxidative synergist of alfa-tocopherol as estimated by hepatic chemiluminescence. Nutrition Reports International 29, 157-165.

Mouri K, Ikesu H, Esaka T \& Igarashi O (1984) The influence of marine oil intake upon levels of lipids, $\alpha$-tocopherol and lipid peroxidation in serum and liver of rats. Journal of Nutritional Science and Vitaminology 30, 307-318.

Nestel PJ (1990) Effects of n-3 fatty acids on lipid metabolism. Annual Review of Nutrition 10, 149-167.

Noguchi T, Cantor AH \& Scott MT (1973) Mode of action of selenium and vitamin $\mathrm{E}$ in prevention of exudative diathesis in chicks. Journal of Nutrition 103, 1502-1511.

Ohkawa H, Ohishi N \& Yagi K (1979) Assay for lipid peroxides in animal tissues by thiobarbituric acid reaction. Analytical Biochemistry 95, 351-358.
Pietrangelo A, Grandi R, Tripodi A, Tomasi A, Ceccarelli D, Ventura E \& Masini A (1990) Lipid composition and fluidity of liver mitochondria, microsomes and plasma membrane of rats with chronic dietary iron overload. Biochemical Pharmacology 39, 123-128.

Rao KS \& Recknagel RO (1968) Early onset of lipoperoxidation in rat liver after carbon tetrachloride administration. Experimental and Molecular Pathology 9, 271-278.

Reitman S \& Frankel S (1957) A Colorimetric method for the determination of serum glutamic oxalacetic and glutamic pyruvic transaminase. American Journal of Clinical Pathology 28, $56-63$.

Roe JH, Mills MB, Oesterling MJ \& Damron CM (1948) The determination of diketo-1-gulonic acid, dehydro-1-ascorbic acid, and 1-ascorbic acid in the same tissue extract by the 2,4-dinitrophenyl-hydrazine method. Journal of Biological Chemistry 174, 201-208.

Saito M (1990) Polychlorinated biphenyls-induced lipid peroxidation as measured by thiobarbituric acid-reactive substances in liver subcellular fractions of rats. Biochimica et Biophysica Acta 1046, 301-308.

Saito M, Kubo K \& Ikegami S (1996) An assessment of docosahexaenoic acid (DHA) intake with special reference to lipid metabolism in rats. Journal of Nutritional Science and Vitaminology 42, 195-207.

Saito M, Nakatsugawa K, Oh-hashi A, Nishimuta M \& Kodama N (1992) Comparison of vitamin E levels in human plasma, red blood cells, and platelets following varying intakes of vitamin E. Journal of Clinical Biochemistry and Nutrition 12, 59-68.

Saito M, Oh-hashi A, Kubota M, Nishide E \& Yamaguchi M (1990) Mixed function oxidases in response to different types of dietary lipids in rats. British Journal of Nutrition 63, 249-257.

Saito M \& Yamaguchi M (1988) Influence of excessive ascorbic acid dose on liver microsomal mixed function oxidase system in guinea pigs. Journal of Clinical Biochemistry and Nutrition 4, 123-137.

Simopoulos AP (1991) Omega-3 fatty acids in health and disease and in growth and development. American Journal of Clinical Nutrition 54, 438-463.

Simopoulos AP (1999) Essential fatty acids in health and chronic disease. American Journal of Clinical Nutrition $\mathbf{7 0}$, 560S-569S.

Song JH, Fujimoto K \& Miyazawa T (2000) Polyunsaturated $(n-3)$ fatty acids susceptible to peroxidation are increased in plasma and tissue lipids of rats fed docosahexaenoic acidcontaining oils. Journal of Nutrition 130, 3028-3033.

Tappel AL (1962) Vitamin E as the biological lipid antioxidant. Vitamins and Hormones 20, 493-510.

Tsuchida M, Miura T, Mizutani K \& Aibara K (1985) Fluorescent substances in mouse and human sera as a parameter of in vivo lipid peroxidation. Biochimica et Biophysica Acta 834, 196-204.

Visioli F, Colombo C \& Galli C (1998) Oxidation of individual fatty acids yields different profiles of oxidation markers. Biochemical and Biophysical Research Communications 245, 487-489.

Wells WW, Xu DP \& Washburn MP (1995) Glutathione: dehydroascorbate oxidoreductases. Methods in Enzymology 252, 30-38.

Winkler BS, Orselli SM \& Rex TS (1994) The redox couple between glutathione and ascorbic acid: a chemical and physiological perspective. Free Radical Biology and Medicine 17, $333-349$.

Yagi K (1976) A simple fluorometric assay for lipoperoxide in blood plasma. Biochemical Medicine 15, 212-216. 